\title{
A collocation method for solving integral equations
}

\author{
A.G. Ramm \\ Mathematics Department, \\ Kansas State University, \\ Manhattan, KS 66506-2602, USA \\ E-mail: ramm@math.ksu.edu \\ Website: http://www.math.ksu.edu/ ramm
}

\begin{abstract}
A collocation method is formulated and justified for numerical solution of the Fredholm second-kind integral equations. As an application the Lippmann-Schwinger equation is considered. The results obtained provide an error estimate and a justification of the limiting procedure used in the earlier papers by the author, dealing with many-body scattering problems in the case of small scatterers, and with creating materials with a desired refraction coefficient Ramm, A.G. 'Many-body wave scattering by small bodies and applications', J. Math. Phys., Vol. 48, No. 10, 103511; Ramm, A.G. (2008) 'Wave scattering by many small particles embedded in a medium', Phys. Lett. A, Vol. 372, No. 17, pp.3064-3070.
\end{abstract}

Keywords: collocation method; integral equations.

Reference to this paper should be made as follows: Ramm, A.G. (2009) 'A collocation method for solving integral equations', Int. J. Computing Science and Mathematics, Vol.

Biographical notes: Alexander G. Ramm is the Author of more than 570 papers, 12 monographs, two patents. He has given dozens of plenary talks at various Conferences and many lectures at various Universities throughout the world. He was distinguished Foreign Professor in Mexico and Egypt, CNRS Research Professor, a London Mathematical Society lecturer, Khwarizmi Award winner, distinguished HKSTAM speaker, PACOM7 plenary speaker. His areas of research include analysis, applied mathematics, numerical analysis, inverse problems, wave scattering, signal estimation, random fields estimation, wave scattering by small bodies, tomography, inverse scattering problems, etc.

\section{Introduction}

In a Banach space $X=L^{\infty}(D), D \subset \mathbb{R}^{3}$ is a bounded domain, consider an equation

$$
A u:=(I+T) u=f, \quad T u=\int_{D} T(x, y) u(y) d y,
$$

Copyright (C) 2009 Inderscience Enterprises Ltd. 
where $I$ is the identity operator and $T$ is a linear compact operator. We assume that $\mathcal{N}(A):=\{u: A u=0\}=\{0\}$. Then $A$ is a bijection of $X$ onto $X$ and $A^{-1}$ is bounded.

Equation (1) with $f=u_{0}:=e^{i k \alpha \cdot x}$, where $\alpha$ is a given unit vector, the direction of the incident plane wave, and $T u:=\int_{R^{3}} \frac{e^{i k|x-y|}}{4 \pi|x-y|} q(y) u(y) d y$, is the well-known Lippmann-Schwinger equation for the scattering solution $u$. The motivation for this paper came mainly from the author's work (Ramm, 2007d, 2008) on wave scattering by many small bodies. It is proved in Ramm (2007d) that (1) is the limiting version of the linear algebraic system, derived in Ramm (2007d). The result of this paper can be considered as a rigorous derivation of the error of the approximation of the solution to (1) by the solution of the linear algebraic system, derived in Ramm (2007d). A similar linear algebraic system was used also in Ramm (2008), and our result is applicable to this system as well.

Let

$$
\sup _{x \in D} \int_{D}|T(x, y)| d y:=N<\infty .
$$

Consider a partition of $D$ by small cubes $D_{j}$ with a side $\frac{1}{n}$,

$$
D \subset \cup_{j=1}^{j_{n}} D_{j}, \quad \operatorname{diam} D_{j}:=d_{n}=\frac{\sqrt{3}}{n},
$$

where $j_{n}=O\left(n^{3}\right)$ is the number of the partition cubes.

Denote by $x_{j}$ the centre of $D_{j}$, by $\chi_{j}(x)$ the characteristic function of $D_{j}$,

$$
\chi_{j}(x)= \begin{cases}1 & \text { in } D_{j} \\ 0 & \text { in } D_{j}^{\prime}:=D / D_{j} .\end{cases}
$$

By $\omega_{u}\left(\frac{1}{n}\right)$ we denote the continuity modulus of $u$,

$$
\omega_{u}(\delta):=\sup _{|x-y| \leq \delta, x, y \in D}|u(x)-u(y)| .
$$

If $u \in C^{a}(D):=\operatorname{Lip}_{a}(D), 0<a<1$, then $\omega_{u}(\delta) \leq c \delta^{a}$. Consider the equation in $\mathbb{R}^{j_{n}}$ :

$$
u_{i}+\sum_{j=1}^{j_{n}} T_{i j} u_{j}=f_{i}, \quad 1 \leq i \leq j_{n} .
$$

Here

$$
u_{i}:=u\left(x_{i}\right), \quad T_{i j}:=\int_{D_{j}} T\left(x_{i}, y\right) d y, \quad f_{i}:=f\left(x_{i}\right) .
$$

Equation (3) is in one-to-one correspondence with the following equation in $X$ :

$$
u_{i}(x)+\sum_{j=1}^{j_{n}} \chi_{i}(x) \int_{D_{j}} T\left(x_{i}, y\right) u_{j}(y) d y=f_{i}(x), \quad 1 \leq i \leq j_{n},
$$

where

$$
u_{i}(x)=u_{i} \chi_{i}(x) .
$$


Define

$$
u^{(n)}(x):=\sum_{i=1}^{j_{n}} u_{i} \chi_{i}(x)
$$

Equation (5) is equivalent to the following one:

$$
\left(I+T_{n}\right) u^{(n)}:=u^{(n)}(x)+\int_{D} T^{(n)}(x, y) u^{(n)}(y) d y=f^{(n)}(x),
$$

where

$$
T^{(n)}(x, y):=\sum_{i=1}^{j_{n}} \chi_{i}(x) T\left(x_{i}, y\right)
$$

Lemma 1: Equation (8) in $X$ is equivalent to equation (3) in $\mathbb{R}^{j_{n}}$ in the following sense: If $\left\{u_{i}\right\}_{i=1}^{j_{n}}$ solves (3), then function (7) solves (8), and, conversely, if function (7) solves (8), then $\left\{u_{i}\right\}_{i=1}^{j_{n}}$ solves (3).

Proof: Let $\left\{u_{i}\right\}_{i=1}^{j_{n}}$ solves (3). Multiply (3) by $\chi_{i}(x)$ and sum up over $i$ from 1 to $j_{n}$ to get (8) with $T^{(n)}$ defined in (9).

Conversely, if $u^{(n)}$, defined in (7), solves (8), then, setting in (7) $x=x_{i}$ one gets the numbers $u_{i}$, because $\chi_{j}\left(x_{i}\right)=\delta_{i j}=\left\{\begin{array}{l}0 \text { if } i \neq j \\ 1 \text { if } i=j\end{array}\right.$. Setting in (8) $x=x_{i}$, one gets (3) with $T_{i j}$ defined in (4). Lemma 1 is proved.

Let us make the following assumption:

Assumption A: The operator $I+T$ is injective, $T$ is compact in $X$, and

$$
\max _{x \in D} \int_{D}\left|\nabla_{x} T(x, y)\right| d y:=c_{0}<\infty .
$$

Our main result is the following.

Theorem 2: If Assumption A holds, then for all sufficiently large $n$, say $n>n_{0}$, system (3) has a unique solution and

$$
\left\|u^{(n)}(x)-u(x)\right\| \leq c \omega_{u}\left(\frac{1}{n}\right) \longrightarrow 0, \quad \text { as } n \rightarrow \infty
$$

where $u^{(n)}$ is defined in (7).

In Section 2, Theorem 2 is proved. Collocation method, proposed in this paper for solving equation (1), does not depend on the choice of the partition of $D$ or the choice of the collocation nodes $x_{i} \in D_{i}$. Different versions of the collocation method were discussed in the literature. Originally a version of this method was proposed by L.V. Kantorovich in 1934 Kantorovich (1934), its generalisation 
can be found in Kantorovich and Akilov (1980) and its version is discussed in Krasnoselskii et al. (1972). The version, presented in our paper, is closely related to the results in the author's earlier papers Ramm (2007d, 2008).

Remark 1: Our results and the method remain valid if the operator $I+T$ in (1) is replaced by $B+T$, where $B$ is an isomorphism of $X$ onto $X$.

Remark 2: The collocation method (3) can be used for solving integral equation of the first kind. The author hopes to give more in a separate paper. The idea is to use the Dynamical Systems Method (DSM) developed in Ramm (2004, 2005a, 2005b, 2005c, 2006a, 2006b, 2007a, 2007b, 2007c) for solving ill-posed problems, and applied specifically to solving ill-conditioned linear algebraic systems in Hoang and Ramm (2008a, 2008b, 2009, 2010) and Ramm (2009). At the end of Section 2 an example of an integral equation for the scattering solution (the Lippmann-Schwinger equation) is discussed. It is checked that the collocation method of Section 1 is applicable to this equation.

\section{Proof of Theorem 1}

The idea of the proof is simple: first we check that

$$
\lim _{n \rightarrow \infty}\left\|T_{n}-T\right\|=0
$$

This and assumption A) imply that the operator $I+T_{n}$ is boundedly invertible for all sufficiently large $n$, say $n>n_{0}$, that is, $I+T_{n}$ is a continuous bijection of $X$ onto $X$. Thus, $\mathcal{N}\left(I+T_{n}\right)=\{0\}$. By Lemma 1 equation (8) is equivalent to (3). Therefore, the homogeneous equation (3) has only the trivial solution for $n>n_{0}$, so, by the Fredholm alternative, equation (3) is solvable for $n>n_{0}$. Its solution generates the function $u^{(n)}(x)$ by formula (7).

To prove (10), one uses the following estimates

$$
\begin{aligned}
\left\|u^{(n)}-u\right\| & =\left\|\left(I+T_{n}\right)^{-1} f_{n}-(I+T)^{-1} f\right\| \\
& \leq\left\|(I+T)^{-1}\right\|\left\|f_{n}-f\right\|+\left\|\left(I+T_{n}\right)^{-1}-(I+T)^{-1}\right\|\left\|f_{n}\right\| \\
& \leq c\left(\left\|f_{n}-f\right\|+\left\|\left(I+T_{n}\right)^{-1}-(I+T)^{-1}\right\|\right) .
\end{aligned}
$$

If $f \in C^{a}(D), 0<a \leq 1$, then

$$
\left\|f-f_{n}\right\| \leq \omega_{f}\left(\frac{1}{n^{a}}\right) .
$$

In applications, for instance, in the example below, one often may assume that $a=1$. In this case

$$
\left\|f-f_{n}\right\| \leq \frac{c}{n} \text { as } n \rightarrow \infty .
$$

In general, one has $\omega_{u}(\delta) \leq c \omega_{f}(\delta)$, if the operator $(I+T)^{-1}$ is bounded and the kernel of $T$ is sufficiently smooth, for example, satisfies Assumption A. 
Let us assume that

$$
\max _{x \in D} \int_{D}\left|\nabla_{x} T(x, y)\right| d y:=c_{0}<\infty .
$$

Then one has

$$
\begin{aligned}
\left\|T-T_{n}\right\| & =\sup _{x \in D} \int_{D}\left|T^{(n)}(x, y)-T(x, y)\right| d y \leq \max _{i, x \in D_{i}} \int_{D}\left|T^{(n)}(x, y)-T(x, y)\right| d y \\
& \leq d_{n} \max _{x \in D} \int_{D}\left|\nabla_{x} T(x, y)\right| d y \leq c_{0} d_{n} \leq \frac{c}{n} .
\end{aligned}
$$

One may replace (14) by a less restrictive assumption

$$
\lim _{n \rightarrow \infty} \sup _{x} \int_{D}\left|T^{(n)}(x, y)-T(x, y)\right| d y=0 .
$$

From (15) one concludes that (11) holds if (14) is assumed, and the rate of convergence is specified in (15) by the factor $d_{n}=\frac{\sqrt{3}}{n}$. If instead of (14) less is assumed, namely,

$$
\lim _{x \rightarrow \xi} \int_{D}|T(x, y)-T(\xi, y)| d y=0,
$$

then (11) holds, but the rate of convergence is not specified. Theorem 2 is proved.

Remark 3: The usual collocation method can be described as follows. Let $X_{n} \subset X$ be a sequence of linear subspaces, $\operatorname{dim} X_{n}=n, X_{n} \subset X_{n+1}$, which is limit-dense in $X$, i.e., for any $u \in X$ one has $\lim _{n \rightarrow \infty} \operatorname{dist}\left(u, X_{n}\right)=0$. Let $\left\{w_{i}(x)\right\}_{i=1}^{n}$ be a basis of $X_{n}$, and the points $x_{j} \in D, 1 \leq j \leq n$, are chosen so that

$$
\operatorname{det} w_{i}\left(x_{j}\right) \neq 0 .
$$

Then the linear system

$$
\sum_{i=1}^{n} c_{i} w_{i}\left(x_{j}\right)=f_{j}, \quad 1 \leq j \leq n,
$$

is uniquely solvable for any $f_{j}$.

An approximate solution $u_{n}(x)$ of equation (1) is sought of the form

$$
u_{n}(x)=\sum_{j=1}^{n} c_{j} w_{j}(x)
$$

and the constant coefficients $c_{j}$ are to be found from the system

$$
\sum_{j=1}^{n} c_{j} w_{j}\left(x_{i}\right)+\sum_{j=1}^{n} c_{j} \int_{D} T\left(x_{i}, y\right) w_{j}(y) d y=f\left(x_{i}\right), \quad 1 \leq i \leq n .
$$


In this scheme, in contrast with our scheme, the choice of the points $x_{j}$ is subject to the condition (18). As the basis function $w_{j}(x)$ one often takes polynomials or trigonometric polynomials. In this case the projection operator $P_{n}$ in $C(D)$ on the subspace of polynomials of degree $\leq n$ has a norm which tends to infinity as $n \rightarrow \infty$. In our version of the collocation method $w_{j}(x)=\chi_{j}(x)$ and $\chi_{j}\left(x_{i}\right)=\delta_{i j}$, so condition (18) holds for any choice of $x_{i} \in D_{i}$. Moreover $P_{n}$, the projection operator onto $X_{n}=\operatorname{span}\left\{\chi_{1}(x), \ldots, \chi_{n}(x)\right\}$ is uniformly bounded by the Banach-Steinhaus Theorem since $P_{n} \rightarrow I$, where $\rightarrow$ denotes strong convergence.

In place of $\left\{\chi_{j}(x)\right\}_{j=1}^{j_{n}}$ one may use linear or higher order splines in $D_{j}$. In this case additional difficulties arise, namely, computing multidimensional splines in the cubic subdomains, and the choice of the collocation points. The higher order splines give a better approximation of smooth functions, but one may not have a smooth solution to an integral equation, and the gain in the accuracy of the approximation may be not compensating the complications due to the construction of multidimensional splines corresponding to the chosen partition of $D$.

Remark 4: If the kernel $T(x, y)$ is unbounded at some set, for example, on the diagonal $x=y$, but $\sup _{x \in D} \int_{D}|T(x, y)| d y<\infty$, then our method is still valid.

Remark 5: General necessary and sufficient conditions for convergence of projection methods (see, e.g., Krasnoselskii et al., 1972; Ramm, 1986, p.152) can be applied for a study of the convergence of collocation methods.

Example: Consider the integral equation for the scattering solution:

$$
u(x)+\int_{D} g(x, y) q(y) u(y) d y=f(x)
$$

where $D \subset \mathbb{R}^{3}$ is a bounded domain,

$$
g(x, y):=\frac{e^{i k|x-y|}}{4 \pi|x-y|}, \quad k=\mathrm{const}>0, \quad f(x)=e^{i k \alpha \cdot x}
$$

$\alpha \in S^{2}$ is a given unit vector and $q(x) \in L^{\infty}(D)$ is a real-valued given function. It is proved in Ramm (2007d) that if $q(x) \in L^{2}(D), I m q \leq 0$, then equation (21) has a unique solution in $H^{2}(D)$, where $H^{2}(D)$ is the usual Sobolev space. Since $f$ is differentiable (in fact $f \in C^{\infty}(D)$ ), one has $\omega_{f}\left(\frac{1}{n}\right)=O\left(\frac{1}{n}\right)$. In our example $T(x, y):=g(x, y) q(y)$, and one has:

$$
\int_{D}\left|\nabla_{x} g(x, y)\right||q(y)| d y \leq c
$$

Thus, assumption (14) holds, Theorem 2 is applicable, and equation (21) can be solved by the convergent collocation method (3). If $D \subset \mathbb{R}^{3}$ and $u \in H^{2}(D)$, then the Sobolev embedding theorem implies that $u \in C^{a}(D), 0<a<\frac{1}{2}$. Thus, in this case, one has $\omega_{u}\left(\frac{1}{n}\right)=O\left(\frac{1}{n^{a}}\right)$. 


\section{References}

Hoang, N.S. and Ramm, A.G. (2008a) 'Solving ill-conditioned linear algebraic systems by the dynamical systems method', Inverse Probl. in Sci. and Engineering, Vol. 16, No. 5, pp.617-630.

Hoang, N.S. and Ramm, A.G. (2008b) 'An iterative scheme for solving equations with monotone operators', BIT, Vol. 48, No. 4, pp.725-741.

Hoang, N.S. and Ramm, A.G. (2009) 'Dynamical systems method for solving finite-rank operator equations', Ann. Polon. Math., Vol. 95, No. 1, pp.77-93.

Hoang, N.S. and Ramm, A.G. (2010) 'Dynamical systems method for solving nonlinear equations with monotone operators', Math. Comp., Vol. 79, No. 269.

Kantorovich, L.V. (1934) 'On a new method for solving partial differential equations', Doklady Acad. Sci. USSR, Vol. 2, Nos. 8-9, pp.532-536.

Krasnoselskii, M.A., Vainikko, G., Zabreiko, P., Rutitskii, Ya. and Stetsenko, V. (1972) Approximate Solutions of Operator Equations, Wolters-Noordhoff, Groningen.

Kantorovich, L.V. and Akilov, G.P. (1980) Functional Analysis, Pergamon Press, New York.

Ramm, A.G. (1986) Scattering by Obstacles, D. Reidel, Dordrecht.

Ramm, A.G. (2004) 'Dynamical systems method for solving operator equations', Communic. in Nonlinear Sci. and Numer. Simulation, Vol. 9, No. 2, pp.383-402.

Ramm, A.G. (2005a) 'Dynamical systems method (DSM) and nonlinear problems', in Lopez-Gomez, J. (Ed.): Spectral Theory and Nonlinear Analysis, World Scientific Publishers, Singapore, pp.201-228.

Ramm, A.G. (2005b) 'DSM for ill-posed equations with monotone operators', Comm. in Nonlinear Sci. and Numer. Simulation, Vol. 10, No. 8, pp.935-940.

Ramm, A.G. (2005c) 'Discrepancy principle for the dynamical systems method', Communic. in Nonlinear Sci. and Numer. Simulation, Vol. 10, No. 1, pp.95-101.

Ramm, A.G. (2006a) 'Dynamical systems method for nonlinear equations in Banach spaces', Communic. in Nonlinear Sci. and Numer. Simulation, Vol. 11, No. 3, pp.306-310.

Ramm, A.G. (2006b) 'Dynamical systems method and a homeomorphism theorem', Amer. Math. Monthly, Vol. 113, No. 10, pp.928-933.

Ramm, A.G. (2007a) Dynamical Systems Method for Solving Operator Equations, Elsevier, Amsterdam.

Ramm, A.G. (2007b) 'Ill-posed problems with unbounded operators', Journ. Math. Anal. Appl., Vol. 325, pp.490-495.

Ramm, A.G. (2007c) 'Iterative solution of linear equations with unbounded operators', Jour. Math. Anal. Appl., Vol. 330, No. 2, pp.1338-1346.

Ramm, A.G. (2007d) 'Many-body wave scattering by small bodies and applications', Jour. Math. Phys., Vol. 48, No. 10, 103511.

Ramm, A.G. (2008) 'Wave scattering by many small particles embedded in a medium', Phys. Lett. A, Vol. 372, No. 17, pp.3064-3070.

Ramm, A.G. (2009) 'Dynamical systems method for solving linear ill-posed problems', Ann. Polon. Math., Vol. 95, No. 3, pp.253-272. 